\title{
Movimento de consolidação nacional de novas bases para o Ensino de Geociências
}

\author{
Carlos Alberto Lobão Cunha \\ Instituto de Geociências, Universidade \\ Estadual de Campinas, Campinas, SP. \\ lobao@ige unicamp.br
}

\begin{abstract}
NATIONAL CONSOLIDATION MOVEMENT TOWARDS NEW BASES FOR GEOSCIENCES EDUCATION. This contribution of the event "Interfaces Geosciences and Education: 40 years of experience (1973-2013)" has divided the concerns of the geological community in regard to educational issues into three distinct phases. Specifically for the second phase of this process it describes recommendations from the community, which have been decisive for production of a Minimal Curriculum for the Geology courses. The set of guidelines on Teaching of Geology in Brazil can be summarized as follows: (1) the ideal profile of a Geologist, as defined during preceding events (in Camboriu/SC and Belo Horizonte/ $M G$ ), is the basic parameter for changes in Geology Teaching; (2) any form of specialization courses in Geology should be avoided; (3) field activities are essential for the formation of a geologist and should lead Teaching Geology from the beginning to end of a course, (4) MEC is asked to fund the activities of the Eschwege Geology Centre due to its well-known importance at the national level; (5) an evaluation of teaching by teachers and students of Geology should be constant; (6) the organizations related to this sector should discuss ways to evaluate and monitor Teaching of Geology; (7) the geological community repudiates the governmental educational policy, as the privatization of public universities, and argues MEC to maintain research and teaching in these institutions; (8) the geological community believes that there should be ongoing discussion in schools about full implementation of the curriculum and teaching methodology; (9) the Geology courses should discuss and contribute to deeper democratization in Brazilian universities. Citation: Cunha C.A.L.S. 2014. Movimento de consolidação nacional de novas bases para 0 Ensino de Geociências. Terræ Didatica, 10(3):171-177. http://www.ige.unicamp.br/terraedidatica/.
\end{abstract}

KEYWORDS : Curriculum guidelines, curricula, higher education, Geology, university, professional regulation.

RESUMO Este artigo do evento "Interfaces Geociências e Ensino: 40 anos de experiências (1973-2013)" divide em três fases distintas as manifestações das preocupações da comunidade geológica em relação a questões educacionais. $O$ trabalho situa o envolvimento e a participação organizada da comunidade na segunda fase desse longo processo, da qual resultaram recomendações determinantes para o ensino de graduação. O conjunto de orientações sobre o Ensino de Geologia no Brasil pode ser sintetizado da seguinte forma: (1) recomenda-se o perfil ideal do geólogo definido durante os eventos de Camboriú/SC e o Simpósio de Belo Horizonte/MG como parâmetro básico para mudanças no Ensino de Geologia; (2) deve-se evitar qualquer forma de especialização nos cursos de Geologia; (3) as atividades de campo são essenciais para a formação do geólogo e devem centralizar o Ensino de Geologia, do início ao final do curso; (4) solicita-se ao MEC o custeio das atividades do Centro de Geologia Eschwege; (5) deve haver constante avaliação do ensino pelos professores e alunos dos cursos de Geologia; (6) as entidades do setor devem discutir formas para avaliar e acompanhar o Ensino de Geologia; (7) a comunidade geológica repudia a política educacional do governo, de privatização das Universidades Públicas, $e$ defende que cabe ao MEC manter a pesquisa e o ensino nessas instituições; (8) a comunidade geológica entende que deve haver discussão permanente nas escolas sobre implementação do currículo pleno e metodologia do ensino; (9) os cursos de Geologia devem discutir e contribuir para profunda democratização nas universidades brasileiras.

PALAVRAS-CHAVE: Currículo mínimo, Educação Superior, Geologia, universidade, regulamentação profissional, mudança curricular. 


\section{Preliminares}

As manifestações das preocupações da comunidade geológica para com suas questões educacionais podem ser divididas em três fases distintas se considerarmos sob o ponto de vista de envolvimento e participação organizada.

A primeira fase tem seu registro inicial no I Encontro de Geólogos realizado em Porto Alegre/ RS, em março de 1966, quando foi levantado, entre outros temas, o debate sobre o ensino. Nos Anais do evento ficou registrada a inquietação com a possibilidade de especializações na graduação, com a criação da pós-graduação e com bolsas de estudo para ambos os níveis. Em agosto de 1974, os estudantes da Escola de Minas Ouro Preto, por meio da Sociedade de Intercâmbio Cultural e Estudos Geológicos (SICEG), promoveram a sua XV Semana de Estudos, tendo como um dos dois temas centrais do encontro $O$ ensino de geologia no Brasil que contou com o magnífico reitor da Universidade Federal de Ouro Preto na abertura e a participação do diretor do Departamento de Assuntos Universitários (DAU) do MEC como um dos conferencistas. Deve ser destacada a apresentação do trabalho dos professores Elmer Prata Salomão e Eduardo Antonio Ladeira, da Universidade de Brasília $(\mathrm{UnB})$, que tratava da experiência pedagógica de integração das disciplinas Geologia Estrutural e Estratigrafia e isso era uma enorme novidade.

Em 1976, em Ouro Preto/MG, o XXIX Congresso Brasileiro de Geologia, conforme já havia acontecido no XXVIII, em Porto Alegre/RS, incluiu no seu Programa novamente uma Mesa Redonda sobre ensino. Eram preocupações protocolares que giravam em torno de algumas comunicações dos coordenadores de cursos. Para não nos deixar esquecer que estávamos em plena ditadura, circulavam nos corredores, com crachás da comissão organizadora, pessoas suspeitas de pertencerem aos órgãos de segurança. Essas observações foram feitas por geólogos que tentavam reorganizar as associações profissionais, como a Coordenação Nacional dos Geólogos (CONAGE). De 1971 a 1974, por iniciativas de entidades profissionais, foram publicadas três pesquisas voltadas para as dificuldades antevistas com o mercado de trabalho para geólogos (ver: UFBa/ABG 1971, Silva 1972 e Souza 1973/74).

Passada a perplexidade dos primeiros anos, em 1978, no XXX Congresso Brasileiro de Geologia, realizado em Recife/PE, na sua já permanente
"Mesa Redonda sobre Ensino", ensaiou-se um levantamento das condições do ensino de Geologia no país com a participação dos diversos coordenadores de cursos. Nessa Mesa foi encaminhada à diretoria da SBG a solicitação para que colocasse em atividade a Comissão de Ensino, conforme constava de seus estatutos. Estava iniciada a segunda fase.

Em março de 1979, em reunião do seu Conselho Diretor, a SBG implantou a Comissão Nacional de Ensino (CNE), com o primeiro e principal objetivo realizar o diagnóstico do ensino de Geologia recomendado no Congresso de Recife. Antes da iniciativa se concretizar, as suas validade e oportunidade foram ainda confirmadas em duas ocasiões. A primeira, em setembro de 1979, em Cuiabál MT, durante o Encontro Nacional de Estudantes de Geologia (ENEGEO), em que, após amplos debates, o setor estudantil aderiu formalmente à idéia de profunda avaliação do ensino de geologia. A segunda ocasião foi durante o II Simpósio Regional de Geologia realizado em Rio Claro/SP, em novembro de 1979.

Em novembro de 1979 a CNE da SBG iniciou formalmente suas atividades, com o objetivo já definido de realizar o diagnóstico do ensino de geologia no País. O projeto foi ao encontro das diretrizes delineadas pelo Ministério da Educação e Cultura, através de sua Secretaria para o Ensino Superior (SESu), que forneceu o apoio financeiro e material necessário para a execução. A pesquisa diagnóstica A formação do geólogo nas universidades brasileiras - um retrato de duas décadas foi levada a efeito pela SBG com todo o apoio do MEC. Iniciada em dezembro de 1979, concluída em maio de 1981, e publicada oficialmente em outubro do próprio ano de 1981, essa pesquisa demarca os trabalhos em relação ao ensino de geologia no Brasil.

Durante o XXXI CBG, em outubro de 1980, a Mesa Redonda de Ensino, realizada em Camboriú/ $\mathrm{SC}$, apresentou, em relação às Mesas e Congressos anteriores, um significativo avanço. Isso pode ser avaliado tanto pelo aumento quantitativo (aproximadamente 500 participantes), como qualitativo (apresentação de Teses para debate), da participação em três Sessões muito concorridas. Os próprios presentes em Camboriú, porém, perceberam a necessidade de aprimorar alguns aspectos dessa crescente participação. Entre esses aspectos, destacavam-se: evitar que as decisões tivessem caráter regional; não permitir a predominância de um ou outro setor da categoria sobre os demais; garantir os canais de participação a todos os setores. Os 
principais saldos dessa Mesa Redonda foram (1) as contribuições para a definição do perfil ideal do geólogo brasileiro que, após a coleta de dados e opiniões junto aos profissionais, foi definido com maior clareza e incorporado às conclusões finais da própria Pesquisa, (2) a definição de assumir radicalmente o caráter deliberativo de suas próprias decisões, em especial em sua Plenária Final e (3) a decisão de realizar um simpósio específico para tratar das questões de ensino.

Deve ser destacado o importante papel desempenhado, em todo o processo, pela Sub-Comissão de Ensino do Núcleo de São Paulo da SBG.

\section{Os Simpósios de Ensino}

A diretoria da SBG assumiu a realização do Simpósio de Ensino e aceitou a oferta da Geologia da Universidade Federal de Minas Gerais. Podemos distinguir quatro etapas prévias ao Simpósio de Belo Horizonte.

1a etapa- Preparação das Teses: Toda a Comunidade foi convocada, através da Circular 1, para, a coletiva ou individualmente, contribuir com as reflexões e propostas, consubstanciadas em forma de Tese. Nessa etapa, uma edição preliminar do Documento final da Pesquisa SBG/MEC foi distribuiudo como subsídio a todas as Entidades. O período destinado a essa etapa foi de três meses (maio a julho) e o resultado foram 26 Teses, elaboradas por 83 autores, editadas em dois volumes.

2a etapa- Discussões das Teses: Essa etapa foi explicitada por avisos e instruções contidas na Circular 2. Os Volumes de Teses foram enviados a todas as Entidades e a todos os autores. A Comissão Temática do Simpósio visitou pessoalmente a maioria dos Cursos de Geologia. Núcleos da SBG e Entidades Profissionais, mantendo contatos e proferindo palestras, mobilizando e esclarecendo diversos setores para as discussões prévias em torno das Teses. Essa etapa desenvolveu-se basicamente em setembro e na primeira quinzena de outubro e delas emergiram propostas alternativas às veiculadas pelas Teses.

3a etapa- Escolha dos Delegados: A Circular 3 , entre outras coisas, divulgou o Regulamento de Escolha de Delegados ao Simpósio. Tal escolha ocorreu no âmbito de cada Entidade Profissional, cada corpo discente e cada corpo docente, fundamentalmente durante a segunda quinzena de setembro. Essa etapa representou a culminância das duas anteriores, na medida em que a participação na elaboração das Teses e nas discussões prévias, foi um dos principais critérios utilizados pelos diversos setores para a escolha de seus delegados.

4a etapa- Preparação dos Delegados: No início de outubro a quarta e última Circular divulgou a lista quase completa dos delegados. Simultaneamente, durante a primeira quinzena de outubro, iniciou-se a preparação dos delegados para levarem ao Simpósio as posições e sugestões alternativas dos diversos setores representados.

Em Belo Horizonte estiveram presentes cerca de 350 participantes, incluindo delegados e não delegados (Ver tabela 1). O total de delegados credenciados foi de 81 (71\% das vagas previstas), sendo 32 docentes ( $84 \%$ dos previstos), 33 estudantes ( $87 \%$ dos previstos) e 16 profissionais ( $42 \%$ dos previstos). O corpo docente esteve representado por delegados de 18 cursos (95\% dos existentes); o corpo discente esteve representado por delegados de 17 cursos (89\%); o corpo de profissionais esteve representado por delegados de 8 Associações (62\% das existentes), estando ausentes os profissionais de 5 outras associações.

As principais conclusões do simpósio se deram a partir do temário efetivamente cumprido: pontos críticos do ensino de geologia no Brasil; metodologia de ensino e avaliação; currículo e condições de funcionamento dos cursos de geologia; formação, aperfeiçoamento e desempenho docente; aspectos do mercado de trabalho e das políticas mineral, econômica, científico-tecnológica e educacional, que interferem na formação do geólogo.

Dentre o grande número de resoluções de natureza variada (posicionamentos, recomendações e medidas de implementação), tomadas em relação aos temas mencionados, foi possível destacar conjuntos individualizados e sintetizados de conclusões que deverão guiar os passos da Comunidade Geológica mostrando como ela encara a sua questão educacional, conforme alguns pontos a seguir apresentados:

a. - O perfil ideal do Geólogo Brasileiro, tal como foi definido por intermédio de pesquisa $A$ formação do geólogo nas universidades brasileiras - um retrato de duas décadas, realizada pela SBG/MEC, deve ser o parâmetro básico para todas as formulações referentes ao ensino de Geologia. Conforme a seguir: "(1) Deverá ter uma formação polivalente voltada para as necessidades do País na área de Geologia, que o capacite para enfrentar, muitas vezes sozinho, 
Tabela 1. Delegados credenciados presentes ao primeiro simpósio (81), discriminados por tipo de representação e tipo de entidade representada, 1981 , com totais

\begin{tabular}{|c|c|c|c|c|}
\hline \multicolumn{3}{|c|}{ UNIVERSIDADES } & \multicolumn{2}{|c|}{ ENTIDADES PROFISSIONAIS } \\
\hline & Delegados Docentes & Delegados Discentes & & $\begin{array}{l}\text { Deleg. Prof. fora da } \\
\text { Univ. }\end{array}$ \\
\hline FUAM & 2 & 2 & APROGAM & - \\
\hline UFPA & 2 & 2 & APGAM & 3 \\
\hline UNIFOR & 1 & 2 & \multirow{2}{*}{ AGEC } & \multirow{2}{*}{-} \\
\hline UFCE & 1 & 2 & & \\
\hline \multirow{2}{*}{ UFPE } & \multirow{2}{*}{2} & \multirow{2}{*}{1} & AGP & 1 \\
\hline & & & AGESE & _ \\
\hline UFBa & 2 & 2 & $\mathrm{ABG}$ & 1 \\
\hline UFRRJ & 2 & 2 & \multirow{3}{*}{ APRJ } & \multirow{3}{*}{1} \\
\hline UFRJ & 2 & 2 & & \\
\hline UERJ & 2 & 2 & & \\
\hline USP & 1 & 2 & \multirow{2}{*}{ SIGESP } & \multirow{2}{*}{3} \\
\hline UNESP & 2 & 2 & & \\
\hline UFPR & 2 & 2 & AGEPAR & - \\
\hline URFS & 2 & 2 & \multirow[b]{2}{*}{ APSG } & \multirow[b]{2}{*}{1} \\
\hline UNISINOS & 1 & - & & \\
\hline UFMG & 2 & 2 & \multirow{2}{*}{ APMIG } & \multirow{2}{*}{3} \\
\hline UFOP & 2 & 2 & & \\
\hline UnB & - & 2 & \multirow{2}{*}{ AGECO } & \multirow{2}{*}{3} \\
\hline UFMT & 2 & 2 & & \\
\hline UFRN & 2 & - & AGERN & - \\
\hline
\end{tabular}

trabalhos de campo em áreas diversificadas e problemas de natureza diversa. (2) Deverá ter uma formação científica que o capacite a desenvolver atividades profissionais com caráter inovador e não somente habilitá-lo para a simples aplicação de metodologias estabelecidas em outros países, muitas vezes inadequadas à nossa realidade. (3) Deverá ter uma postura crítica perante sua atuação profissional e perante os principais problemas que afetam os setores estratégicos da economia nacional. (4) Deverá ter uma formação independente das diretrizes governamentais com características imediatistas, obedecendo a um planejamento que retrate a projeção de necessidades do país previstas a médio e longo prazo. (5) Deverá ser um profissional com participação social e política junto a entidades de classe e outras agremiações políticas."

b. - A Política Mineral adotada pelo Governo, em relação ao Projeto Grande Carajás, deve ser rejeitada, pois foi implantada sem qualquer consulta à sociedade brasileira como um todo e, em particular, à Comunidade Geológica. A exploração dos recursos minerais da região de Carajás vem privilegiando os grandes grupos multinacionais, em detrimento das necessidades brasileiras, inclusive por não utilizar o potencial cientifico e tecnológico nacional. 
Tabela 2. Delegados credenciados presentes ao segundo simpósio (59), discriminados por tipo de representação e tipo de entidade representada, 1982, com totais.

\begin{tabular}{|c|c|c|c|c|c|c|c|c|}
\hline \multicolumn{3}{|c|}{ Universidades } & \multicolumn{2}{|c|}{ Entidades profissionais } & \multicolumn{4}{|c|}{ Núcleos regionais da S.B.G. } \\
\hline & $\begin{array}{l}\text { Delegados } \\
\text { Docentes } \star\end{array}$ & $\begin{array}{c}\text { Delegados } \\
\text { Discentes } \\
\star \star\end{array}$ & & $\begin{array}{c}\text { Delegados } \\
\text { Profissionais } \\
\star \star \star \\
\end{array}$ & & $\begin{array}{c}\text { Delegados } \\
\text { Docentes }\end{array}$ & $\begin{array}{c}\text { Delegados } \\
\text { Discentes }\end{array}$ & $\begin{array}{l}\text { Delegados } \\
\text { Profissionais }\end{array}$ \\
\hline FUAM & 1 & - & APROGAM & - & MANAUS & - & - & - \\
\hline UFPA & 1 & 1 & APGAM & - & NORTE & 1 & 1 & - \\
\hline UNIFOR & 1 & 1 & \multirow{2}{*}{ AGEC } & \multirow{2}{*}{-} & \multirow{2}{*}{ FORTALEZA } & \multirow{2}{*}{-} & \multirow{2}{*}{-} & \multirow{2}{*}{-} \\
\hline UFCE & 1 & 1 & & & & & & \\
\hline UFRN & 1 & 1 & AGERN & - & \multirow{3}{*}{ NORDESTE } & \multirow{3}{*}{-} & \multirow{3}{*}{1} & \multirow{3}{*}{ - } \\
\hline \multirow[t]{2}{*}{ UFPE } & 1 & 1 & AGEP & - & & & & \\
\hline & & & AGESE & - & & & & \\
\hline UFBa & 1 & 1 & $\mathrm{ABG}$ & & BAHIA & 1 & 1 & 1 \\
\hline UFMG & 1 & 1 & \multirow{2}{*}{ APMIG } & & \multirow{2}{*}{$\begin{array}{l}\text { MINAS } \\
\text { GERAIS }\end{array}$} & \multirow{2}{*}{1} & \multirow{2}{*}{1} & \multirow{2}{*}{1} \\
\hline UFOP & 1 & 1 & & & & & & \\
\hline UFMT & - & 1 & \multirow[t]{2}{*}{ AGECO } & & $\begin{array}{c}\text { CENTRO- } \\
\text { OESTE }\end{array}$ & - & - & 1 \\
\hline UnB & 1 & 1 & & & BRASÍLIA & - & - & - \\
\hline UFRRJ & - & 1 & \multirow{3}{*}{ APRJ } & & \multirow{3}{*}{$\begin{array}{c}\text { RIO DE } \\
\text { JANEIRO }\end{array}$} & \multirow{3}{*}{1} & \multirow{3}{*}{1} & \multirow{3}{*}{1} \\
\hline UFRJ & 1 & 1 & & & & & & \\
\hline UERJ & 1 & 1 & & & & & & \\
\hline USP & 1 & 1 & \multirow{2}{*}{ SIGESP } & & \multirow{3}{*}{ SÃO PAULO } & \multirow{3}{*}{1} & \multirow{3}{*}{1} & \multirow{3}{*}{1} \\
\hline UNESP & 1 & 1 & & & & & & \\
\hline UFPR & - & 1 & AGEPAR & - & & & & \\
\hline
\end{tabular}

Notas: * Escolhidos pelas Direções das respectivas Unidades Universitárias. ** Escolhidos pelos respectivos Centros ou Diretórios Acadêmicos. ${ }^{* * *}$ Algumas Entidades Profissionais, para atender à representação paritária das três categorias, puderam escolher mais de um delegado, segundo critérios de proporcionalidade estabelecidos pela CONAGE

c. - O ensino deve ser público e gratuito. A autonomia universitária deve ser mantida. A Universidade deve se democratizar, inclusive no seu plano interno, promovendo a escolha de seus dirigentes através de processos com a participação de todos os setores.

d. - A Comunidade Geológica deve se posicionar previamente em relação a todas as iniciativas referentes à relação empresa-escola. $\mathrm{Na}$ análise das situações concretas, devem ser levados em consideração a autonomia universitária e o mencionado perfil ideal do geólogo. Por violar estes dois princípios, a Comunidade Geológica se posiciona contra o GEOPET, curso promovido pela Petrobrás no último ano do Curso de Geologia da Universidade Federal da Bahia.

e. - Em cada curso de Geologia deve ser criado um Centro para atuar junto à Sociedade. Tal Centro deve trabalhar na resolução de problemas sociais (que necessitem da intervenção dos geólogos).

f. - A Comunidade Geológica precisa se envolver no amplo debate de âmbito nacional que visa redefinir o papel das escolas de $1^{\circ} \mathrm{e}$ $2^{\circ}$ graus.

g. - A metodologia do ensino de Geologia deve sofrer profundas reformulações, tendo em vista uma maior e mais crítica participação dos estudantes no processo de aprendizagem e uma maior coerência com o perfil ideal do geólogo. [...]

h. - Deve ser dada prioridade, seja no nível da SBG, CONAGE e ENEGE, seja no nível das instituições universitárias, às estruturas e mecanismos que possibilitem a efetiva implementação das mudanças educacionais preconizadas. Esses mecanismos e estruturas devem ser voltados tanto para o 
amadurecimento do significado das decisões do Simpósio, como para a melhoria da participação conjunta de professores e alunos no encaminhamento das diversas questões educacionais.

Durante o II Simpósio sobre Ensino de Geologia no Brasil, realizado em setembro de 1982, no XXXII Congresso Brasileiro de Geologia, em Salvador/BA, foi aprovada a proposta da comunidade geológica para o novo currículo mínimo da graduação.

Diferentemente do I Simpósio, e por solicitação da própria diretoria da SBG, parte dos delegados foi escolhida pela sociedade por meio de seus Núcleos Regionais. A paridade entre os três universos foi mantida mas o atendimento das pressões da SBG implicou a redução do número máximo de delegados para noventa (Ver tabela 2).

Durante os três dias de realização do Simpósio, com 59 delegados, ocorreram dois Painéis, duas Sessões Plenárias, diversas Sessões de Debates, além das Sessões de Abertura e Encerramento e das sessões de credenciamento e elaboração de Pauta num total de setenta e duas horas de trabalho quase ininterrupto. Foram exaustivamente examinadas as nove Teses previamente distribuídas para a Comunidade Geológica.

O currículo mínimo aprovado diferia profundamente dos anteriores, tanto em seu conteúdo como na forma da sua elaboração: os conteúdos de abrangência das matérias são muito mais explícitos; a classificação das matérias emerge de uma classificação das ciências geológicas, segundo uma base epistemológica e psico-pedagógica previamente definida; a proposta curricular resulta de um amplo e longo processo de discussões e decisões em que foram abertos canais efetivos de participação para todos os segmentos da comunidade geológica.

Das deliberações aprovadas emergiu um conjunto de orientações sobre o Ensino de Geologia no Brasil:

1- Foi referendado que o perfil ideal do geólogo é o parâmetro básico para as mudanças no Ensino de Geologia, conforme definido no Simpósio de Belo Horizonte;

2- Os cursos de graduação em Geologia devem evitar qualquer forma de especialização;

3- As atividades de campo são essenciais para a formação do geólogo; elas devem ser o centro de todo o Ensino de Geologia, desde o início até o final do curso;
4- O Centro de Geologia Eschwege desempenha um papel fundamental na formação dos geólogos, porém esse papel não vem sendo considerado nas dotações orçamentárias. Há uma tentativa do MEC no sentido do fechamento desse centro de pesquisa e ensino através da diminuição de seus recursos. A Comunidade Geológica solicita ao MEC que custeie as atividades do Centro de Geologia Eschwege;

5- Os professores e alunos dos cursos de Geologia devem constantemente avaliar o ensino. Devem-se montar coordenadorias Interdisciplinares para ser esse instrumento de avaliação, conforme Resolução 36 do Simpósio de Belo Horizonte;

6- A SBG, CONAGE, ENEGE e Associações Docentes devem, conjuntamente, discutir as formas para avaliar e acompanhar o Ensino de Geologia;

7- A comunidade geológica repudia a política educacional do governo, de privatização das Universidades Públicas e defende que cabe ao MEC manter a pesquisa e o ensino nessas instituições;

8- A comunidade geológica entende que a implementação do currículo pleno e a metodologia do ensino devem ser uma discussão permanente nas escolas;

9- Os cursos de Geologia devem discutir e contribuir constantemente para uma profunda democratização nas universidades brasileiras. Conforme a documentação examinada destaca-se que há reiteradas manifestações (desde 1966) contra a especialização na graduação e $\boldsymbol{a}$ favor de uma formação polivalente voltada para as necessidades do País na área de Geologia. Tal manifestação foi consolidada no Perfil ideal do geólogo - Mesa redonda de Camboriú/SC, 1980, Pesquisa SBG/ MEC, 1981, e ratificada nos dois Simpósios.

Ao longo do processo, a grande mobilização e participação de todos os setores (docentes, discentes e profissionais não atuantes na universidade) garantiram o mesmo peso de decisão nos Simpósios de Ensino, assim como ficou patente que a percepção das questões educacionais foi, e deve ser, vista de modo bastante amplo.

Entre 1979 e 1982 foram produzidos, e amplamente divulgados, os seguintes documentos:

a. Pesquisa SBG/MEC - versão preliminar e versão final;

b. Vinte e seis Teses ao Simpósio de Belo Horizonte - dois volumes; 
c. Documento síntese do I Simpósio;

d. Documento final do I Simpósio;

e. Nove Teses ao Simpósio de Salvador;

f. Documento final do II Simpósio.

A partir daí temos a terceira fase quando, após toda essa mobilização e participação coletiva, a comunidade geológica incorporou as atividades de educação em Mesas Redondas aos Congressos da SBG, mas agora com uma nova qualidade de participação e enfrentamento dos problemas.

\section{Referências Bibliográficas}

Amaral I.A.do, Manfredi S.M., Negrão O.B.M. 1981. A formação do geólogo nas universidades brasileiras : um retrato de duas décadas. São Paulo: Soc. Bras. Geol. e Min. Educ. Cultura. 209p.

Cunha C.A.L. 1995. Geologia introdutória nas instituições de ensino superior no Brasil : análise dos cursos de ciências e geografia. Campinas: Fac. Educ., Univ. Est. Campinas. 268p. (Tese Dout. Educ.).

Primeiro Encontro de Geólogos, 1966, Porto Alegre. Anais... Porto Alegre: Cons. Nac. Pesq. e Univ. Fed. Rio Grande do Sul. 272p.
Semanas de Estudo, XIV., XV, 1973, 1974. 1978. Ouro Preto. Anais... Ouro Preto: Sociedade de Intercâmbio Cultural e Estudos Geológicos. 295p.

Silva A.M.M. 1972. Geologia : estudos e perspectivas de trabalho. São Paulo: Fund. Carlos Chagas. 82p.

Sociedade Brasileira de Geologia (SBG). 1982. Simpósio Nacional sobre o Ensino de Geologia no Brasil, 1. 1981 Belo Horizonte. Documento Final... São Paulo: Sociedade Brasileira de Geologia. 155p.

Sociedade Brasileira de Geologia (SBG). 1983. Simpósio Nacional sobre o Ensino de Geologia no Brasil. Currículo Mínimo, 2. 1982, Salvador. Documento Final... São Paulo: SBG. 73p.

Sociedade Brasileira de Geologia (SBG). 1984. A formação do geólogo nas universidades brasileiras: um retrato de duas décadas. São Paulo: SBG.

Souza J.A.de. 1973/74. Pesquisa nacional sobre o mercado de trabalho de geólogos e engenheiros de minas. Belo Horizonte: Min. Educ. Cult. e Univ. Fed. Minas Gerais. 2v.

Universidade Federal da Bahia, Associação Baiana de Geólogos. 1971. Caracterização sócio-econômica e mercado de trabalho do geólogo. Salvador: Univ. Fed. Bahia e Assoc. Baiana Geól. 140p. 